\title{
No-reference image quality assessment through the von Mises distribution
}

\author{
Salvador Gabarda and Gabriel Cristóbal* \\ Instituto de Óptica (CSIC), Serrano 121, 28006 Madrid, Spain \\ *Corresponding author: gabriel@optica.csic.es
}

Received February 17, 2012; revised June 28, 2012; accepted July 24, 2012; posted August 9, 2012 (Doc. ID 163213); published September 6, 2012

\begin{abstract}
An innovative way of calculating the von Mises distribution of image entropy is introduced in this paper. The von Mises distribution's concentration parameter and some fitness parameter that will be defined later have been analyzed in the experimental part for determining their suitability as an image quality assessment measure in some particular distortions such as Gaussian blur or additive Gaussian noise. To achieve such measure, the local Rényi entropy is calculated in four equally spaced orientations and used to determine the parameters of the von Mises distribution of the image entropy. Considering contextual images, experimental results after applying this model show that the best-in-focus noise-free images are associated with the highest values for the von Mises distribution concentration parameter and the highest approximation of image data to the von Mises distribution model. Our defined von Mises fitness parameter experimentally appears also as a suitable no-reference image quality assessment indicator for no-contextual images. (c) 2012 Optical Society of America
\end{abstract} OCIS codes: $\quad 110.3000,100.2000,330.6180,180.0180$.

\section{INTRODUCTION}

Digital images can suffer a number of different operations that take into account, e.g., acquisition, coding, compression, transmission, and many others before their final use. In such processes, digital images might be affected by many kinds of degradations revealed by different types of visual distortions. A list of different possible types of degradations has been considered in the image database TID2008 by Ponomarenko et al. [1]. A key feature about images is their quality. However, image quality is a concept difficult to define. In many situations it is defined through the degree in which some requirements are fulfilled. In a semantic approximation, image quality is understood as the subjective impression of how well image content is rendered or reproduced [2]. This definition yields to the concept of image quality assessment. In practice, such assessment can be achieved by two means: one is by psychophysical subjective experiments with human observers, and the other one is by objective metrics applied directly to digital images [3]. Image quality assessment is a very active research area with many contributions, techniques, and models [4]. Typically, image quality can be measured by comparison with a reference, but unfortunately such a reference is not available in many applications. Hence, no-reference image quality assessment methods are required, and they have been the subject of very active research during the last years. However, a universal method for estimating the overall image quality is still a challenging open issue. Customarily, existing noreference methods deal with simple specific degradations such as Gaussian blur or additive white noise. A good survey about existing no-reference sharpness metrics may be found in Ferzli and Karam [5] , where their metric is compared to others with good results. Ferzli and Karam introduced the notion of just noticeable blur (JNB). It is an edge-based sharpness metric based on a human visual system model. Other metrics have been developed including blur and noise simultaneously, such as the one by Zhu and Milanfar [6], introducing a new concept called true image content. Their measure is correlated with noise, sharpness, and intensity contrast, manifested in visually salient geometric features such as edges, showing that such a measure correlates well with subjective quality evaluation for both blur and noise distortions. However, the Zhu and Milanfar technique has been designed to compare images within the same context (images covering the same area but having different quality attributes), while the sharpness metric of Ferzli and Karam has been developed to predict the relative amount of blurriness in images regardless of their context (note that, in what follows, we will consider that images resulting from distorting a given original enclose the same context and we will use the term different context for degraded or distorted images resulting from different originals). According to $\mathrm{Zhu}$, the JNB technique fails to capture the trend of quality change in block-matching and threedimensional (BM3D) [6] denoising experiments, since it cannot handle noise well. Later on, Narvekar and Karam [7] proposed an improved algorithm based on the JNB paradigm for a noreference objective image sharpness metric, introducing a technique they called the cumulative probability of blur detection (CPBD). In this work, the sharpness metric converges to a finite number of quality classes. They used the LIVE [8] database to validate the performance of their metric. A training-based method determines the centroids of the quality classes that represent the perceived quality levels. Classification is based on assigning the image to one of the quality classes and then using the index of the corresponding quality class as the metric value for that image. They include measuring experiments for Gaussian blur and JPEG2000-compressed images, and they show that this metric performs better than other known metrics.

In summary, diverse reference and no-reference classifications of metrics can be considered. According to the type of 
distortion they measure, specific metrics (only one type) versus universal metrics (all types) could be considered. Concerning its contextual character, we can find contextual metrics when comparing images within the same context or no-contextual metrics when they provide a measure regardless of the image context. In addition to the previous schemes, supervised and unsupervised approaches may be considered in the image quality framework.

This paper is a step forward from the approach introduced by Gabarda and Cristóbal [9] that links image directional entropy with image quality assessment. In [9], the variance of the directional entropy of the image was introduced as a quality assessment parameter, presenting the highest value when the image is free of Gaussian blur or Gaussian noise and decreases monotonously with blur and noise increments. Gabarda and Cristóbal's method may be classified as a contextual, no-reference, specific, and unsupervised metric. In this paper, we extend this methodology for image quality assessment to both contextual and no-contextual applications by introducing the von Mises distribution of the directional image entropy as the cornerstone of a new image quality metric for blur and noise. We provide a Matlab implementation of the von Mises distribution-based no-reference quality measure that can be downloaded from [10].

This paper is organized as follows. Section 2 gives the mathematical background required to understand our methodology. Section 3 presents some applications of the von Mises distribution for image quality measurement, along with the performances of this method against the mean opinion score (MOS) of the TID2008 image database and the differential mean opinion score (DMOS) of the LIVE image database. Results are compared with scores from other no-reference quality assessment existing methods. Finally, conclusions are drawn in Section 4 .

\section{DESCRIPTION OF THE METHOD}

\section{A. Directional Entropy}

The information content of images can be measured in different ways. One of the most popular is the entropy. Generally speaking, the measurement of entropy was initially proposed by Shannon [11] as a measure of the information content per symbol, coming from a stochastic information source. It can be considered as a quantifier of the uncertainty or randomness of a signal or, in other words, a measure of the information content of the signal. Given a discrete random variable $X=\left\{x_{1}, x_{2}, \ldots, x_{I}\right\}$, the Shannon entropy of this discrete random variable can be computed as

$$
H(X)=-\sum_{i=1}^{I} P\left(x_{i}\right) \log P\left(x_{i}\right),
$$

where $P\left(x_{i}\right)$ is the probability of event $x_{i}$.

Later, Rényi [12] extended this notion to yield the generalized entropy, whose representation for discrete random variables is

$$
R_{\alpha}(X)=\frac{1}{1-\alpha} \log _{2}\left(\sum_{i=1}^{I} P^{\alpha}\left(x_{i}\right)\right) .
$$

Here $\alpha$ is a real-positive number. It can be shown that the Rényi entropy, as a generalization of the Shannon entropy, reverts to the Shannon entropy when $\alpha \rightarrow 1$.
Measures of entropy may be global, affecting the whole image, or may be localized in each pixel by the use of a sliding window, centered in each pixel, that gathers the neighborhood of that pixel with the desired geometry.

Our proposal is to use a linear array of pixel values, centered in the interest pixel and oriented in a set of desired directions, in order to have a directional measure of entropy at pixel level. To do so, an operational sliding window $L$ is defined, containing the $N+1$ pixels that better fit the ideal line centered in the interest pixel and directed by angle $\theta$. $N$ is required to be an even number to preserve the symmetry of the window.

Better than considering the gray values of the image as input to the entropy calculation, we prefer considering the space-frequency information of the image. Space-frequency representations of a signal open up the possibility of exploring the image information taking into account the position and the local frequency content of the image. One of these spacefrequency representations is the Wigner distribution (WD) [13]. A common analytical framework of space-frequency representations was introduced by Cohen [14]. The WD has been selected due to its excellent properties and because it can be regarded as a masterform distribution function from which the other existing representations can be derived as filtered versions of it [15]. The WD approximation for discrete signals is better known as pseudo-Wigner distribution (PWD). To calculate a local PWD, we will use the following equation:

$$
W(n, k)=2 \sum_{m=-\frac{N}{2}}^{\frac{N}{2}-1} z(n+m) z^{*}(n-m) e^{-i 2 \pi k\left(\frac{2 m}{N}\right)} .
$$

This approximation of the PWD is due to Claasen and Mecklenbräuker [16] and is similar to Brenner's expression [17]. In Eq. (3) the variable $z(n)$ represents the gray value of pixel $n$ in a given image $z$. A directional PWD may be calculated by using Eq. (3) along with a pixel-by-pixel sliding window $D_{\theta}$ to pick up the values $z=z(-N / 2), \ldots, z(0), \ldots, z(N / 2)$. The central position of the window matches exactly pixel $n$ on the image. Equation (3) can be interpreted as the discrete Fourier transform (DFT) of the product $r(n, m)=$ $z(n+m) z^{*}(n-m)$. Here $z^{*}$ indicates the complex-conjugate of signal $z$ (note that $z=z^{*}$ for real-valued images). The sum is limited to the $N$ terms whose values are taken in the spatial interval $(-N / 2, N / 2-1)$. In Eq. (3), $n$ and $k$ represent the space and frequency discrete variables, respectively, and $m$ is a shifting parameter, which is also discrete. By shifting the window to all possible positions over the image, the full pixelwise PWD of the image is produced.

In what follows, we will take the PWD in Eq. (3) as the distribution to be used as input to Eq. (2). Consequently, each spatial position $n$ will receive a certain value of entropy.

Diverse measures of entropy may be defined upon the kind of distribution and normalization used with the formulation due to Rényi. Although the Rényi measures in time-frequency distributions formally resemble the original entropies, they do not have the same properties, conclusions, and results derived in classical information theory. The positivity, $P(n, k) \geq 0$, will not be always preserved, along with the unity energy condition $\sum_{k} P(n, k)=1$ for a given pixel $n$. In order to reduce a distribution to the unity signal energy case, some kind of 
normalization should be applied. The normalization can be performed in various ways, leading to a variety of possible measurement definitions [18-22].

The Rényi entropy measure applied to a discrete spacefrequency distribution, as the PWD denoted by $W(n, k)$, can be written as

$$
R(n)=-\frac{1}{2} \log _{2}\left(\sum_{k} W^{3}(n, k)\right)
$$

In [20], it is stated that $\alpha=3$ is the first value to yield a welldefined, useful information measure for time-frequency representations. Along with all Rényi entropies of odd orders, $\alpha=3$ possesses an asymptotic invariance to cross-components in the time-frequency plane that neutralize the contribution of such components in the resulting entropy. Other odd numbers can be used to calculate Rényi entropy, but here $\alpha=3$ has been selected for simplicity, giving Eq. (4). The summation is restricted to the one-dimensional (1D) neighborhood of pixel $n$, as described above. Again $k$ is the frequency discrete variable. In order to reduce the distribution $W(n, k)$ to the unity signal energy case, some kind of normalization must be done [18]. To do so, the PWD given by Eq. (3) is normalized by applying the following normalizing law, as a previous step to the Rényi entropy measure:

$$
\tilde{W}(n, k)=\frac{W(n, k) W^{*}(n, k)}{\sum_{k}\left(W(n, k) W^{*}(n, k)\right)}=\frac{W^{2}(n, k)}{\sum_{k} W^{2}(n, k)} .
$$

Squaring $W$ is a straightforward method for converting it in a probability distribution, provided that the PWD is a realvalued time-frequency representation (only real-positive or real-negative values are possible), and then negative values are converted in positive by squaring. We have tested other normalizations and our experiments have shown that this normalization outperforms the results due to other known normalizations [22]. The normalizing step affects exclusively index $k$, when the window operation in Eq. (4) is applied to pixel $n$; hence the condition $\sum_{k} \tilde{W}(n, k)=1$ in such position is fulfilled.

It can be shown that the Rényi entropy meets the constraint $0 \leq R_{\alpha}(n) \leq \log _{2} N$ [23,24]. Hence, the measure can be normalized to the interval $[0,1]$ by applying $R(n)=R(n) / \log _{2} N$ in Eq. (4).

The $1 \mathrm{D}$ window used to measure entropy by this method may be oriented in any desired direction $\theta$, as indicated above, and entropy is now denoted by $R(n, \theta)$. This local pixelwise directional entropy can be constrained to a scalar value for the whole image by averaging all the $R(n, \theta)$ values by means of

$$
\bar{R}(\theta)=\langle R(n, \theta)\rangle=\frac{1}{M} \sum_{n} R(n, \theta)
$$

provided that $M$ is the number of pixels in the image.

\section{B. Von Mises Distribution}

The von Mises distribution belongs to probability theory and is used to handle directional statistics for continuous probability distribution on a circular basis. It appears, in many respects, analogous to the normal distributions for a scalar variable. It was proposed by von Mises [25] to study deviations of atomic weights from integer values. This distribution has also been applied to diverse applications in many fields and has become an important tool in the statistical theory of directional data [26].

The von Mises probability density function for the angular variable $\theta$ is given by

$$
f(\theta \mid \mu, \kappa)=\frac{1}{2 \pi I_{0}(\kappa)} e^{\kappa \cos (\theta-\mu)}
$$

where $I_{0}(\kappa)$ is the modified Bessel function of order 0 , and $\theta$ is defined in the interval $[-\pi, \pi)$. The parameter $\kappa$ is responsible for how concentrated the distribution is around the mean direction $\mu$. Larger values of the concentration parameter $\kappa$ indicate that the distribution is more closely grouped around the mean direction. When $\kappa=0$, this distribution is equivalent to the uniform distribution. This fact happens when directions in the image are endowed with equal probability. The uniform circular distribution is, for example, a good model for Gaussian noise.

Different authors have dedicated some research to the link between images and the von Mises distribution. For instance, Vo and Oraintara [27] proposed a new statistical modeling of natural images in the wavelet transform domain. They claimed that the von Mises distribution fits accurately the behaviors of relative phases in the complex directional wavelet sub-band from different natural images, and introduced a new image feature based on the von Mises model for image texture retrieval applications. Palacios et al. [28] presented what they called new tools for color image processing, based on the circularity of the hue variable of a color image. They gave a definition of the median and the range of angular data and applied their results to detect hue edges. Feng [29] presents an image local orientation estimation method, which is based on a combination of two well-known techniques: the principal component analysis (PCA) and the multiscale pyramid decomposition. Grana et al. [30] described a new approach to texture characterization for document analysis. By considering the autocorrelation matrix, they described image texture through a mixture of von Mises distributions.

\section{Von Mises Distribution of Image Entropy}

As a further contribution to the applications cited in the previous section, we propose to use the von Mises distribution for modeling the directional distribution of the image entropy, calculated by means of Eq. (6). The measure defined for the image by Eq. (6) requires the use of a bimodal von Mises distribution, provided that $\bar{R}(\theta)=\bar{R}(\theta+\pi)$, as will be shown later in this section. This requirement is fulfilled when we take $a=1 / 2$ and $\mu_{2}=\mu_{1}+\pi$ in the following bimodal expression of the von Mises distribution:

$$
f(\theta \mid \mu, \kappa)=\frac{a}{2 \pi I_{0}(\kappa)} e^{\kappa \cos \left(\theta-\mu_{1}\right)}+\frac{1-a}{2 \pi I_{0}(\kappa)} e^{\kappa \cos \left(\theta-\mu_{2}\right)},
$$

from which we arrive to [31]

$$
f(\theta \mid \mu, \kappa)=\frac{1}{2 \pi I_{0}(\kappa)} \cosh (\kappa \cos (\theta-\mu)) .
$$


Equation. (9) can be considered as the basis for modeling directional statistics of image entropy. One of the features to take into account in the discrete directional model for image entropy based on Eq. (6) is the number of axes that can be defined in the image. Two requirements must to be fulfilled in order to consider that the directional measures, based on Eq. (6), are completely comparable. First, all directions must be measured through windows consisting of the same number of pixels, and second, the span of the windows in all possible directions must have the same length. These two constrains are only possible when using four directions along the axes of a regular octagon, i.e., $\pi / 8,3 \pi / 8,5 \pi / 8,7 \pi / 8$. This assertion is based first on the fact that the PWD of a given array as, for example, $\left[x_{1}, \ldots, x_{N}\right]$, is the same as that of its reverse array $\left[x_{N}, \ldots, x_{1}\right]$ (note that the orientation of these two arrays differ in $\pi$ ). This feature determines that the angular periodicity of the Rényi entropy is $\pi$. Secondly, the discrete character of the image, represented by pixels, determines some constrains in the topology of pixel arrays across the image. For all this, we have to restrict our calculations to $1 \mathrm{D}$ windows directed by axes along an octogonal arrangement of angles. Figure 1 graphically shows the topological equivalence of the four directions proposed in this paper.

The four directionalities $\theta_{i} \in\{\pi / 8,3 \pi / 8,5 \pi / 8,7 \pi / 8\}$ that we have defined are used to calculate four values $\bar{R}\left(\theta_{i}\right)$ of entropy by means of Eq. () that will be referred to as vectors $\mathbf{R}_{i}=\left(R_{i}, \theta_{i}\right)$. These directional entropies can also be represented by means of Cartesian vectors by $\mathbf{R}_{i}=\left(x_{i}, y_{i}\right)^{T}=$ $\left(R_{i} \cos \theta_{i}, R_{i} \sin \theta_{i}\right)^{T}$.

Some definitions for a resultant vector and a mean angular direction in von Mises distribution have been given by [32] and one analysis for modeling circular data may be found in [33].

In our approximation, we first perform a preliminary estimation of $\mu$ by a single value decomposition (SVD) of the directional vectors $\mathbf{R}_{i}$, according to [29], that tackles the image orientation estimation problem through the local image gradient. The singular vector $\mathbf{v}_{i}$ of the matrix

$$
\mathbf{X}=\left(\begin{array}{c}
\mathbf{R}_{1}^{T} \\
\mathbf{R}_{2}^{T} \\
\mathbf{R}_{3}^{T} \\
\mathbf{R}_{4}^{T}
\end{array}\right)=\left(\begin{array}{ccc}
R_{1} \cos \theta_{1} & R_{1} \sin \theta_{1} \\
R_{2} \cos \theta_{2} & R_{2} \sin \theta_{2} \\
R_{3} \cos \theta_{3} & R_{3} \sin \theta_{3} \\
R_{4} \cos \theta_{4} & R_{4} \sin \theta_{4}
\end{array}\right)
$$

corresponding to its largest singular value gives an estimation of the parameter $\hat{\mu}=\arg \left(\mathbf{v}_{1}\right)$.

Also, an initial estimation of $\kappa$ can be achieved by the following approximation due to Dhillon [34]: $\hat{\kappa}=1 / 2(1-\bar{R})$, where $\bar{R}=\left\|\sum_{i=1}^{4} \mathbf{R}_{i}\right\| / 4$.

The second step is a gradient descent algorithm to find a better estimation for $\kappa$. Also, the value of $\mu$ is updated by
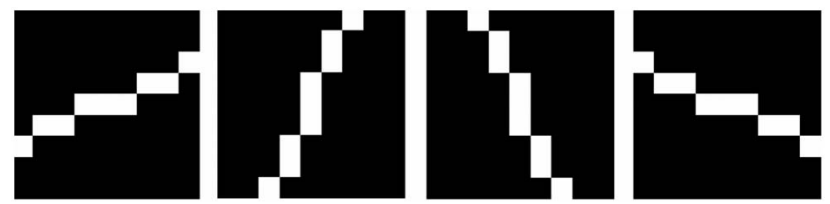

Fig. 1. Different configuration of pixels to define directional windows $D_{\theta}$, with $N=9$ pixels and four equally spaced orientations $\pi / 8,3 \pi / 8,5 \pi / 8,7 \pi / 8$. Note that periodicity is $\pi$, provided that $\theta$ and $\theta+\pi$ originates pixel arrays with the same PWD. determining the angular position of the maximum of the entropy function, after each iteration of $\kappa$. The set of values $\left\{f\left(\theta_{i}\right), R_{i}\right\}$, for $\theta_{i}=\pi / 8,3 \pi / 8,5 \pi / 8,7 \pi / 8$, with $i=1,2,3,4$, respectively, is matched by a minimum square error estimation method to find the coefficients $A$ and $B$ that fit the equation $R_{i}=A f\left(\theta_{i}\right)+B$. An exact fitting is attained if $A=1$ and $B=0$. An error function $\varepsilon(\kappa)=\|(A(\kappa), B(\kappa))-(1,0)\|$ is defined to control the $\kappa$ increments, in order to find a minimum for this error function. The algorithm runs by means of a recursive law $\kappa_{i+1}=(1 \pm C) \times \kappa_{i}, C=0.01$ for updating the values of $\kappa$. The algorithm stops when the error value reaches a minimum. A Matlab implementation of this algorithm may be found in [35].

The accuracy of the estimated distribution $\hat{f}(\theta)$ to a true von Mises distribution is measured by defining a fitness function $\varphi(\varepsilon)=e^{-\varepsilon}$. This function has to be considered here as a measure of the probability that an individual solution survives in the iterative algorithm, as a general law applied in evolutionary computation [36]. Function $\varphi(\varepsilon)$ has been defined by means of an exponential law, in such a way that $0 \leq \varphi \leq 1$, increasing monotonously with decreasing values of $\varepsilon$. The value of $\varphi$ will be 1 for the best fitting and 0 for the worst. The resulting value of $\varphi$ when the iterative algorithm ends is taken as the fitness parameter of the resulting approximation of the von Mises distribution of the image entropy. An example of estimation of the von Mises distribution for a given image is shown in Fig. 2. Triangles indicate the directional entropy values $\left(R_{i}, \theta_{i}\right)$ used as input to the algorithm that estimates the von Mises distribution, $\hat{f}(\theta)$, of the image.

To characterize the behavior of $\kappa$ and $\varphi$, the algorithm has been tested with the images in the image database TID2008 due to Ponomarenko et al. [1]. This database contains 25 reference color images and 1700 distorted images (25 reference images $\times 17$ types of distortion $\times 4$ levels of distortion) in bitmap format without any compression. Images are $512 \times$ 284 pixels in size. The test has been performed taking the 25 reference images, excluding image 25 , provided that this last one is artificial and has been considered inappropriate for our study.

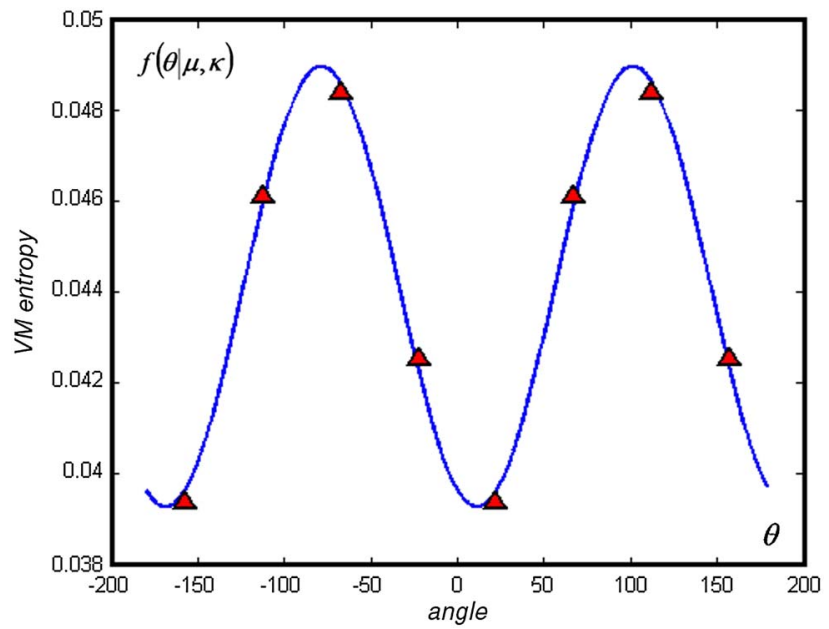

Fig. 2. (Color online) Von Mises distribution of the directional entropy for image I21 contained in the database TID2008 [1]. Triangles indicate the value of directional entropy for the target image after four different axes. $\kappa=-0.36, \mu=-78.75 \mathrm{deg}$ (twin pick at $101.25^{\circ}$ ). Fitness parameter: $\varphi=0.89$. 
Reference images have been first degraded by adding blur in iterative manner by means of a rotationally symmetric Gaussian lowpass filter of size $5 \times 5$ pixels with standard deviation $\sigma=1$, and in a second instance by adding Gaussian noise, also iteratively, with standard deviation $\sigma=0.01$. Considering the 24 originals and nine iterations for each original image, we produced a test set of 240 images. Figures 3 and 4 show the average values of $\kappa$ and $\varphi$ for all these images, having the degradation level, denoted by $\Lambda$, as abscissa. Measurements indicated that $\varphi=0.88 \pm 0.02$ as the expected value of the fitness parameter for the 24 originals (originals are represented by $\Lambda=0$ in images 3 to 8 ), indicating that the fitness parameter $\varphi$ of the von Mises distribution converges to a common value for good-quality images. In general, when more blur or Gaussian noise is added, fitness decreases, and the variance of $\varphi$ increases, indicating that, for severe degradation, the von Mises entropy paradigm for image modeling does not hold anymore.

Figures $\underline{3}-\underline{6}$ show the behavior of the parameters $\kappa$ and $\varphi$ by averaging the values from the outcome of our algorithm for the 24 images in the TID2008 database. A hybrid blur-noise situation has been experimented by adding blur and noise simultaneously to the images in the TID2008 database (see Figs. $\underline{7}$ and $\underline{8}$ ).

Two main consequences are derived from the previous observations. First, the fitness parameter has a low value $(\varphi \ll 0.88)$ for blurred images or very noisy images, and a high value $(\varphi \cong 0.88)$ for undegraded images. The second consequence is that quality of the images is maximum when $\kappa$ reaches a maximum in the series of diversely degraded images from a given original.

\section{APPLICATION OF VON MISES DISTRIBUTION TO IMAGE QUALITY ASSESSMENT}

\section{A. Von Mises Distribution and Image Quality Assessment of Contextual Images}

The behavior of the $\kappa$ parameter of the von Mises distribution suggests that it can be considered as an image quality assessment index, when dealing with Gaussian blur or Gaussian

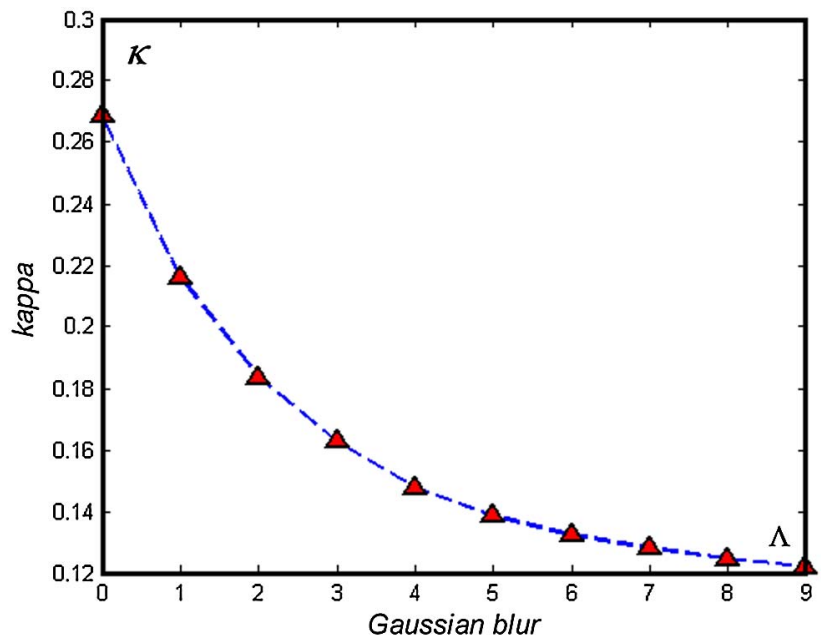

Fig. 3. (Color online) Averaged variation of $\kappa$ (concentration parameter) against increasing amount of Gaussian blur for reference images in the TID2008 database. $\Lambda$ values represent the number of iterations of the blur kernel over the original image.

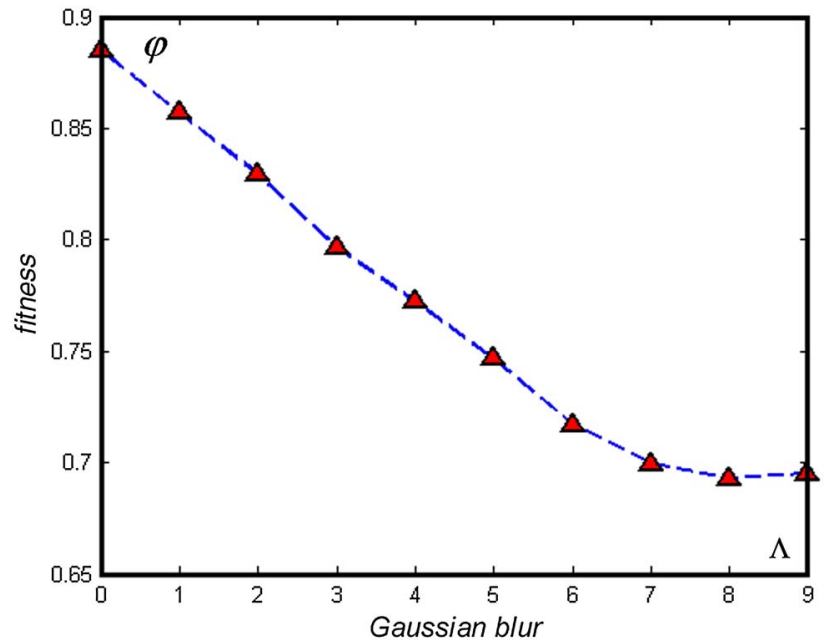

Fig. 4. (Color online) Averaged variation of $\varphi$ (fitness) against increasing amount of Gaussian blur for reference images in the TID2008 database. $\Lambda$ values represent the number of iterations of the blur kernel over the original image.

noise. In order to have a quantitative evaluation of this statement, we compare the classification after this parameter versus the MOS for two specified distortion types in database TID2008.

The image database TID2008 includes 17 different degradation types with one to four strength levels. The image database includes information about the MOS for each distorted image. The degraded images labeled as Gaussian noise and Gaussian blur have been scored by means the $\kappa$ value determined by our von Mises algorithm. Later on, we used the Kendall, Spearman, and Pearson correlation coefficients to evaluate the similarity between the MOS values and the $\kappa$ parameter calculated by means of our method for Gaussian noise and image blur from the TID2008 database. Results are shown in Table 1. Figures in Table 1 must be understood as representing measures in a contextual, specialized, no-reference, and nolearning metric.

In order to put figures in Table 1 in line with other existing no-reference methods, we include Table $\underline{2}$, where our

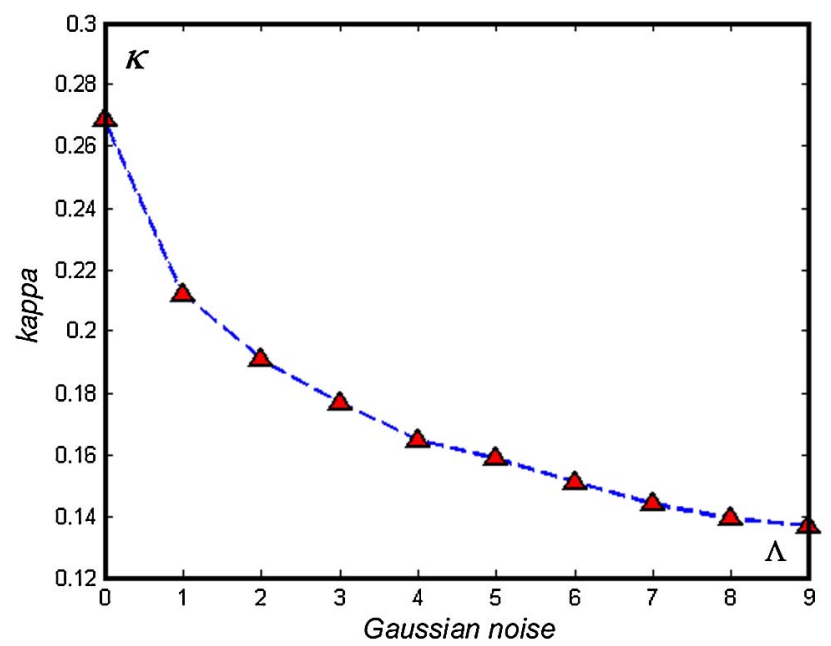

Fig. 5. (Color online) Averaged variation of $\kappa$ (concentration parameter) against increasing amount of Gaussian noise for reference images in the TID2008 database. $\Lambda$ values represent the number of iterations of the noise kernel over the original image. 


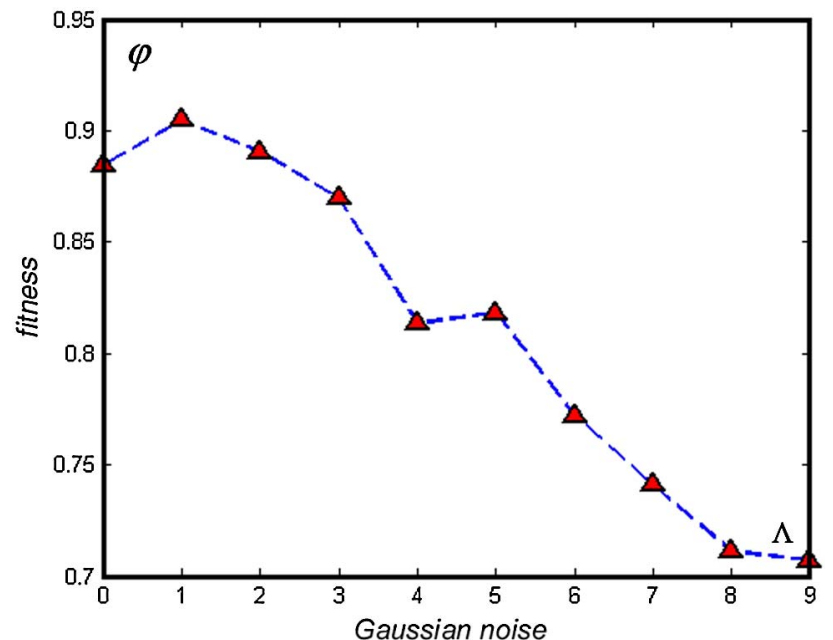

Fig. 6. (Color online) Averaged variation of $\varphi$ (fitness) against increasing amount of Gaussian noise for reference images in the TID2008 database. $\Lambda$ values represent the number of iterations of the noise kernel over the original image.

measures are compared to the results published by Zhu and Milanfar [6] using the same image database and the Spearman correlation coefficient.

Figures in Table 2 reveal that the $\kappa$ parameter of von Mises distribution has a strong correlation with Zhu and Milanfar measures for Gaussian blur and Gaussian noise distortions.

\section{B. Autofocusing Example}

A short depth of field is an inherent limitation in optical microscopy. Basically, the focal plane of the microscope needs to be critically located to observe the sharper image. Hence, autofocusing techniques must be included in automatized optical microscope systems. Relevant examples of images from bright field microscopy may be found in Valdecasas [37].

The behavior of the $\kappa$ parameter of the von Mises distribution suggests that it can be considered as an image quality assessment index, when dealing with Gaussian blur or Gaussian

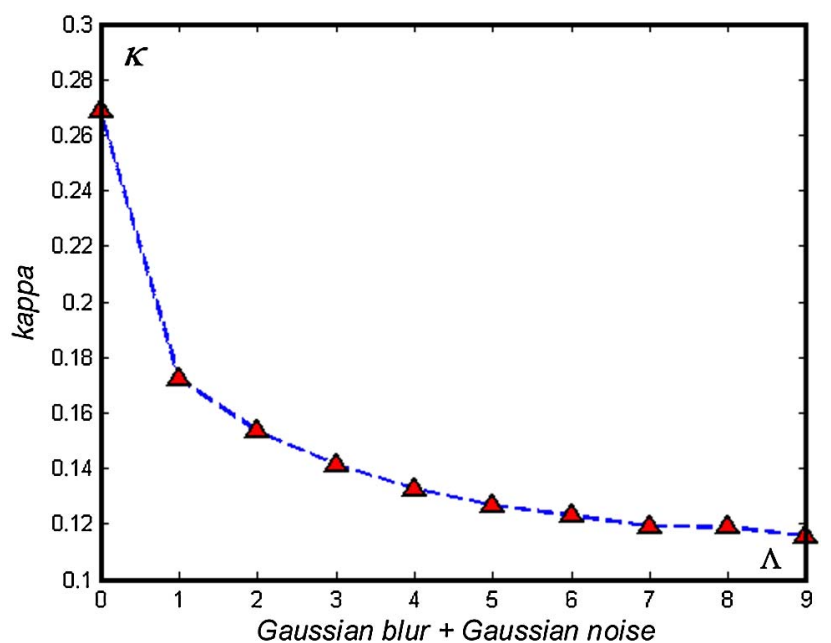

Fig. 7. (Color online) Averaged variation of $\kappa$ (concentration parameter) against increasing amounts of Gaussian blur plus Gaussian noise for reference images in the TID2008 database. $\Lambda$ values represent the number of iterations of the Blur + noise kernel over the original image.

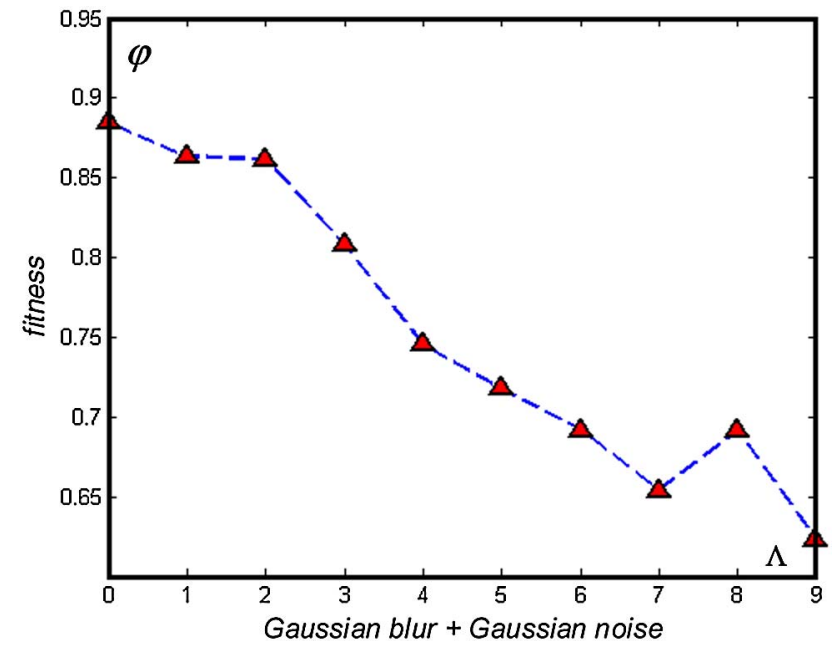

Fig. 8. (Color online) Averaged variation of $\varphi$ (fitness) against increasing amounts of Gaussian blur plus Gaussian noise for reference images in the TID2008 database. $\Lambda$ values represent the number of iterations of the Blur + noise kernel over the original image.

noise. After the above quantitative evaluation of this parameter through the TID2008 database, we present a real-life example to illustrate one of the possible applications of this no-reference image quality assessment method for contextual images. In this example, a sequence of 100 images from an optical microscope has been processed by our algorithm to determine their $\kappa$ values. Images are $256 \times 256$ pixels in size. Images 10, 30, 50, and 70 of the stack are shown in Fig. 9 . The best image according to our algorithm is number 57 in the sequence, which can be seen in Fig. 10. Figure 11 plots the values of $\kappa$ for the whole sequence of images.

\section{Von Mises Distribution and Image Quality Assessment of No-Contextual Images}

One of the objectives of this paper consists in determining whether the von Mises distribution can be used to construct a no-reference quality metric in the no-contextual case. The definition of a metric for image quality assessment based on the von Mises distribution for no-contextual images requires some kind of normalization, to assure independence of the measure from the image context. To do so, we have designed two possible measures taking into account the results in the previous section. Following these results, we postulate that both, the concentration parameter, $\kappa$, and the fitness parameter, $\varphi$, decrease exponentially with increasing amount of degradation (e.g., Gaussian blur), which can be modeled by expressions like $\kappa=\kappa_{0} \exp (-\beta D)$ or $\varphi=$ $\varphi_{0} \exp (-\beta D)$, where $D$ is the degradation and $\beta$ is a shape factor exclusive for each image. Empirically, we have found convergence at the origin for $\varphi_{0}$ to 0.88 , although there is not a similar convergence for $\kappa_{0}$. The second of these expressions is

Table 1. Similarity between $\kappa$ and MOS Values for Images in TID2008, Measured by Kendall, Pearson, and Spearman Coefficients

\begin{tabular}{lccc}
\hline Type of degradation & Kendall & Pearson & Spearman \\
\hline Gaussian noise & 0.7778 & 0.8052 & 0.8083 \\
Gaussian blur & 1.0000 & 0.9600 & 1.0000 \\
\hline
\end{tabular}


Table 2. Spearman Correlation Coefficients for $\kappa$ and Zhu and Milanfar Algorithms [6] Referred to MOS Values in TID2008 Image Database

\begin{tabular}{lccc}
\hline Method & Gaussian blur & Gaussian noise & Blur + noise \\
\hline Zhu and Milanfar & 1.0000 & 0.9760 & 0.9210 \\
$\kappa$ & 1.0000 & 0.8083 & 0.8083 \\
\hline
\end{tabular}

simpler to calculate, provided that $\kappa_{0}$ is an unknown parameter, while $\varphi_{0}$ has been experimentally determined as $\varphi_{0}=$ $0.88 \pm 0.02$ (for Gaussian blur). The exponential variation of $\varphi$ can be written in a logarithmic way as

$$
\log \varphi=\log \varphi_{0}-\beta D
$$

The derivative of Eq. (11) gives $d(\log \varphi) / d D=-\beta$, and $\beta$ can be approximated by

$$
\beta=-\Delta(\log \varphi) / \Delta D
$$

for small increments, $\Delta D$.

Now, let us suppose that an arbitrary image $I_{i}$ produces an outcome parameter $\varphi_{i}$. By convolving the image with an appropriate kernel, $g$ (i.e., a PSF of $5 \times 5$ pixels and $\sigma=1.5$ ), a controlled amount of blur may be added to the image. Then, $I_{i+1}=I_{i} * g$ is a little more degraded image than $I_{i}$. Supposing that the fitness parameter for $I_{i+1}$ is $\varphi_{i+1}$, and making $\Delta D=1$ for a given measuring scale, we can say, from Eq. (12), that $\beta \simeq \log \left(\varphi_{i} / \varphi_{i+1}\right)$, and introducing this value in Eq. (11),

$$
D_{i}=-\left(\log \varphi_{i}-\log \varphi_{0}\right) / \beta \text {. }
$$

Equation (13) defines in this way a new no-contextual measure for image quality assessment for the Gaussian blur case. The measure requires the estimation of the decay constant $\beta$ for each specific image as indicated in Eq. (12). This new measure will be referred hereafter as von Mises degradation measure (VMDM) and $D$ will be expressed in von Mises degradation units.

In order to evaluate the performance of the VMDM, we have applied our algorithm to the Gaussian blur images in the LIVE image database. This group is composed of 144 degraded images plus 29 originals. For our validation test, we have selected a group of 29 images (20\% of the degraded subgroup), i.e., from image number 117 to 145 . Then we have correlated our quality scores with the DMOS scores of this group. Considering that the observations are positive quantities varying over many orders of magnitude, it is plausible to assume that the noise of the observations will be Gaussian, and the data will be well modeled as a Gaussian process. In this context, it is standard practice in the statistics literature to take the log of the data [38]. Then, we have used $\log \left(1+D_{i}\right)$ instead of $D_{i}$ for correlation. Results for the Pearson and Spearman coefficients are shown in Table 3 .

As expected, Table 3 presents good performance results, but is still far from a perfect matching with the DMOS scores. An objective measure must necessarily have some differences when compared with recordings from subjective human observers. Even more, DMOS values will change if the database is created using different methodologies and environmental settings; hence, subjective scores are not comparable [39], while the objective test will remain the same. However, the scores in Table 3 can be improved by applying some weighting transformation to the VMDM scores by incorporating to these scores some learning process that mimics the specific human preferences existing in the DMOS recordings accompanying the LIVE database. To do so, the logarithmic VMDM measures and the transformed VMDM measures may be related, for example, through a function $f$ [38] given by

$$
\begin{aligned}
& \hat{D}=f(D ; \Psi)=D+\sum_{i=1}^{I} a_{i} \tanh \left(b_{i}\left(D+c_{i}\right)\right) \\
& a_{i}, b_{i} \geq 0 \quad \forall i,
\end{aligned}
$$

where $\Psi=\{a, b, c\}$.

Taking $I=5$, the values for the $a_{i}, b_{i}, c_{i}$ coefficients have been determined by a Monte Carlo process that maximizes the Pearson + Spearman correlation with the DMOS scores. We have used images 1 to 116 (the $80 \%$ of the 145 blurred images) in the LIVE database as the learning set and we have used the former set of 29 images for crossvalidating the measures. Our results are shown in Table 4 . The table includes also the results from Narvekar and Karam's method for the same group of distortion.

Comparative results indicate that this method based on the $\varphi$ parameter of the von Mises distribution is highly matching the DMOS scores for the Gaussian blur set of images in the LIVE database. Our results are comparable with the results given by Narvekar and Karam's method, which is based on a sharpness metric for Gaussian blurring when applied to no-contextual images. Our method has the advantage of being self-contained and its performance can be even improved by adding a learning step.

The degradation measure $D$ defined in this section is an alternative method to determine the relative quality of images with different context. Bearing in mind that images with the same context are a special case of images of different context, with context difference equal to zero, the algorithm used in Subsection 3.C is able to work as well for the cases
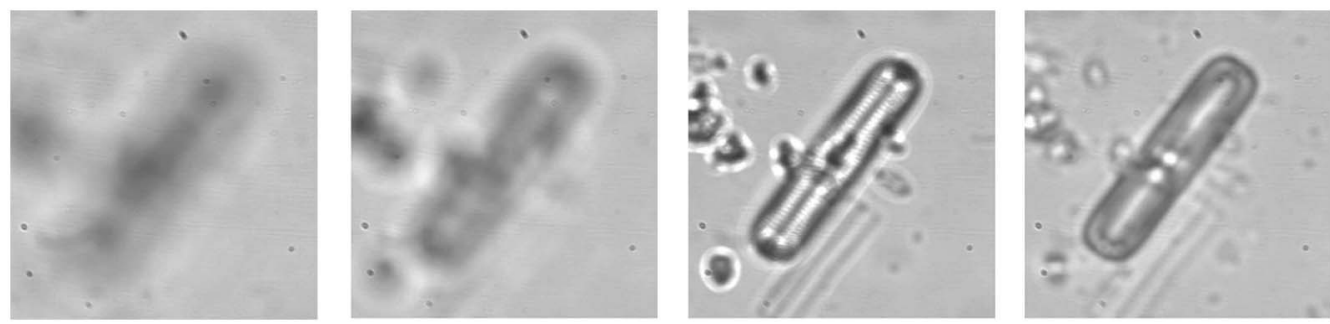

Fig. 9. From left to right, capture 10, 30, 50, and 70 from a sequence of 100 light microscope images. 


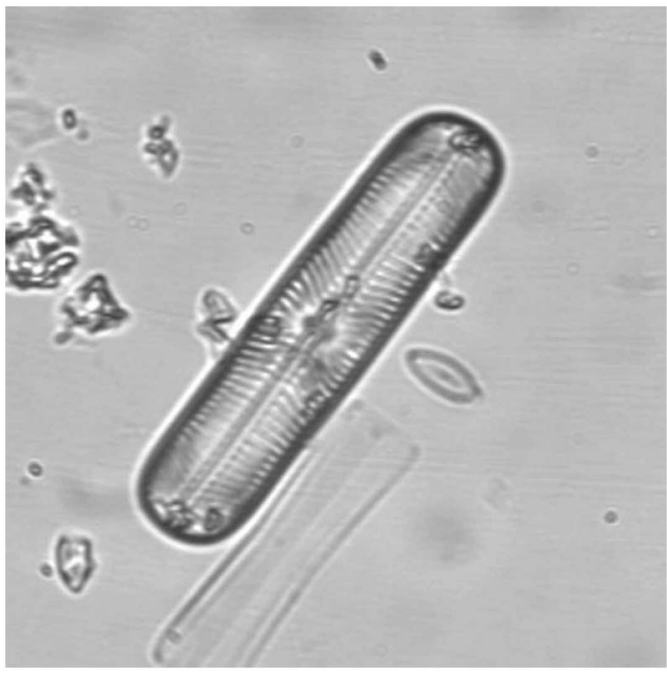

Fig. 10. The best quality image in the sequence (no. 57) after the von Mises algorithm.

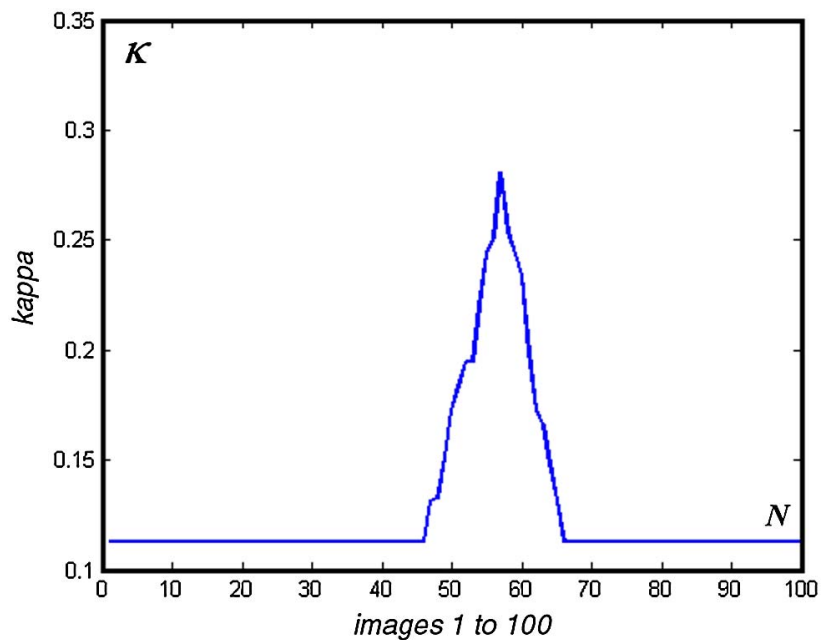

Fig. 11. (Color online) $\kappa$ values for the whole sequence of microscopic images. $N$ represents the position number of images in the sequence. The maximum of $\kappa$ corresponds to image number 57.

considered in Subsections 3.A and 3.B, but the measures will be according with the results obtained in Subsection 3.C. That means that the algorithm used in Subsections 3.A and 3.B should be preferred for such a case and hence, we have declined the evaluation of the VMDM for contextual images. Conversely, the method in Subsections 3.A and 3.B cannot be extended to no-contextual images, and this has been the reason to define measure $D$. The suitability of the exponential relationship between $\varphi$ and $D$ is an ad-hoc conjecture that is in

Table 3. Similarity Between VMDM and DMOS Values for Gaussian Blur in LIVE Database, Measured by Pearson and Spearman Coefficients

\begin{tabular}{cc}
\hline Pearson & Spearman \\
\hline 0.8546 & 0.9275 \\
\hline
\end{tabular}

Table 4. Pearson and Spearman Correlation Coefficients for VMDM and Narvekar and Karam Algorithms Referred to DMOS Gaussian Blur Values in LIVE Image Database

\begin{tabular}{lcc}
\hline Method & Pearson & Spearman \\
\hline Narvekar and Karam & 0.9211 & 0.9449 \\
Transformed VMDM & 0.8926 & 0.9311 \\
\hline
\end{tabular}

agreement with the experiments, but other feasible better approximation through a different function is possible. This is a mathematical optimization problem that is out of the scope of the present paper and that will be considered in future research.

\section{CONCLUSIONS}

In this paper, we have introduced a new way of determining the von Mises distribution of the image information. The possible applications of the von Mises distribution for image quality assessment have been experimentally tested and a set of parameters, $\kappa, \varphi$, and $D$, have been defined as quality assessment indicators for individual images. The $\kappa$ parameter of the von Mises distribution has experimentally shown that it can be considered as a suitable no-reference quality indicator when dealing with contextual images. Significative results have been presented for the Gaussian blur and Gaussian noise cases. Also, the defined von Mises distribution fitness parameter, $\varphi$, has shown its suitability as a no-reference quality indicator in the no-contextual case, but restricted only to Gaussian blur. Not enough significance has been found between $\kappa$ or the fitness parameter $\varphi$ and other types of degradation when dealing with no-contextual images. However, given the strong relationship of the von Mises distribution to the image quality assessment that has been shown in this paper, it is expected that this methodology will provide new applications of the von Mises distribution of image entropy for image processing, and that will be the subject of our further work.

\section{ACKNOWLEDGMENTS}

This work has been partially supported by grants TEC201020307 and TEC2010-09834-E from the Spanish Ministry of Science and Innovation.

\section{REFERENCES}

1. N. Ponomarenko, V. Lukin, A. Zelensky, K. Egiazarian, M. Carli, and F. Battisti, "A database for evaluation of full-reference visual quality assessment metrics," Adv. Mod. Radioelectron. 10 pp. 30-45 (2009).

2. S. Yendrikhovskij, "Image quality and colour categorisation," in W. Lindsay, M. MacDonald, and L. Ronnier, eds., Colour Image Science: Exploiting Digital Media (Wiley, 2002), pp. 393-420.

3. G. Ciocca, F. Marini, and R. Schettini, "Image quality assessment in multimedia applications," Proc. SPIE 7255, 72550A (2009).

4. Z. Wang and A. Bovik, "Modern image quality assessment," in Synthesis Lectures on Image, Video, and Multimedia Processing (Morgan \& Claypool, 2006), Vol. 2, pp. 1-156.

5. R. Ferzli and L. J. Karam, "A no-reference objective image sharpness metric based on the notion of just noticeable blur (JNB)," IEEE Trans. Image Process. 18, 717-728 (2009).

6. X. Zhu and P. Milanfar, "Automatic parameter selection for denoising algorithms using a no-reference measure of image content," IEEE Trans. Image Process. 19, 3116-3132 (2010). 
7. N. D. Narvekar and L. J. Karam, "An improved no-reference sharpness metric based on the probability of blur detection," in Proceedings of International Workshop on Video Processing and Quality Metrics for Consumer Electronics (VPQM), http:// www.vpqm.org [pdf] [Software] (2010).

8. H. R. Sheikh, A. C. Bovik, L. Cormack, and Z. Wang, "LIVE image quality assessment database," http://live.ece.utexas.edu/ research/quality (2003).

9. S. Gabarda and G. Cristóbal, "Blind image quality assessment through anisotropy," J. Opt. Soc. Am. A 24, B42-51 (2007).

10. http://www.iv.optica.csic.es/page49/styled-4/page62.html.

11. C. E. Shannon and W. Weaver, The Mathematical Theory of Communication (University of Illinois, 1949).

12. A. Rényi, "Some fundamental questions of information theory," in Selected Papers of Alfred Rényi (Akadémiai Kiadó, Budapest), Vol. 3, pp. 526-552. (Originally: MTA III. Oszt. Közl., 10, 1960, pp. 251-282) (1976).

13. E. Wigner, "On the quantum correction for thermodynamic equilibrium," Phys. Rev. 40, 749-759 (1932).

14. L. Cohen, "Generalized phase-space distribution functions," J. Math. Phys. 7, 781-786 (1966).

15. L. D. Jacobson and H. Wechsler, "Joint spatial/spatial-frequency representation," Signal Process. 14, 37-68 (1988).

16. T. A. C. M. Claasen and W. F. G. Mecklenbräuker, "The Wigner distribution-a tool for time frequency analysis, parts I-III." Philips J. Res., 35, 217-250 (1980).

17. K. H. Brenner, "A discrete version of the Wigner distribution function," in Proceedings of EURASIP, Signal Processing II: Theories and Applications (1983), pp. 307-309.

18. T. H. Sang and W. J. Williams, "Rényi information and signal dependent optimal kernel design," in Proceedings of the ICASSP (1995), Vol. 2, pp. 997-1000.

19. W. J. Williams, M. L. Brown, and A. O. Hero, "Uncertainty, information and time-frequency distributions," Adv. Signal Process. 1566, 144-156 (1991).

20. P. Flandrin, R. G. Baraniuk, and O. Michel, "Time-frequency complexity and information," in Proceedings of the ICASSP (1994), Vol. 3, pp. 329-332.

21. J. Pitton, P. Loughlin, and L. Atlas, "Positive time-frequency distributions via maximum entropy deconvolution of the evolutionary spectrum," in Proceedings of ICASSP IV (1993), pp. 436-439.

22. L. Stankovic, "A measure of some time-frequency distributions concentration," Signal Process. 81, 621-631 (2001).

23. K. Conrad, "Probability distributions and maximum entropy," Entropy 6, 1-10 (2004).
24. K. Zyczkowski, "Rényi extrapolation of Shannon entropy," Open Syst. Inf. Dyn. 10, 297-310 (2003).

25. R. von Mises "Uber die 'Ganzzahligkeit' der Atomgewicht und verwandte Fragen," Physikalische Z. 19, 490-500 (1918).

26. M. Abramowitz and I. A. Stegun, Handbook of Mathematical Functions (Dover, 1965).

27. A. P. N. Vo and S. Oraintara, "Statistical image modeling using von Mises distribution in the complex directional wavelet domain," in Proceedings of IEEE International Symposium on Circuits and Systems (IEEE, 2008), pp. 2885-2888.

28. A. R. Palacios, C. Rodríguez, and C. Vejarano, "Circular processing of the hue variable a particular trait of colour image processing," in 2nd International Conference on Computer Vision Theory and Applications (VISAPP 2007) (2007), pp. $69-78$.

29. X. Feng, "The analysis and approaches to image local orientation estimation," Master's thesis (University of California, 2003).

30. C. Grana, D. Borghesani, and R. Cucchiara, "Describing texture directions with von Mises distributions," in Proceedings of ICPR (2008), pp. 1-4.

31. M. A. Stephens, "Techniques for directional data," Technical report no. 150 (Department of Statistics, Stanford University, 1969).

32. S. R. Jammalamadaka and A. SenGupta, Topics in Circular Statistics (World Scientific, 2001).

33. J. Bentley, "Modelling circular data using a mixture of Von Mises and uniform distributions" (Department of Statistics and Actuarial Science, Simon Fraser University, 2006).

34. I. S. Dhillon and S. Sra, "Modeling data using directional distributions," Technical Report \# TR-03-06 (Department of Computer Sciences, The University of Texas at Austin, 2003).

35. http://www.mathworks.com/matlabcentral/fileexchange/authors/ 127745 .

36. C. R. Stephens and J. Mora Vargas, "Effective fitness as an alternative paradigm for evolutionary computation I: general formalism," Genet. Program. Evolvable Mach. 1, 363-378 (2000).

37. A. G. Valdecasas, D. Marshall, J. M. Becerra, and J. J. Terrero, "On the extended depth of focus algorithms for bright field microscopy," Micron 32, 559-569 (2001).

38. E. Snelson, C. E. Rasmussen, and Z. Ghahramani, "Warped Gaussian processes," Adv. Neural Inform. Process. Syst. 16 337-344 (2004).

39. J. Redi, R. Zunino, H. Liu, H. Alers, and I. Heynderickx, "Comparing subjective image quality measurement methods for the creation of public databases," Proc. SPIE 7529, 752903 (2010). 